\title{
Microdeletion of the AZFc locus with high frequency of mosaicism 46,XY/47XYY in cases of non obstructive azoospermia in eastern population of India
}

\author{
A.K. Saxena and K. Aniket \\ Department of Pathology/Lab Medicine, Molecular Cytogenetics \\ Laboratory, All India Institute of Medical Sciences, Patna (Bihar), India \\ Corresponding author: A.K. Saxena \\ E-mail: draksaxena1@ rediffmail.com \\ Genet. Mol. Res. 18 (2): gmr18349 \\ Received May 07, 2019 \\ Accepted June 06, 2019 \\ Published June 18, 2019 \\ DOI http://dx.doi.org/10.4238/gmr18349
}

\begin{abstract}
The etiopathology of male infertility is highly complex, involving gene - environment interactions to regulate spermatogenesis. Consequently, genetic analysis becomes imperative for cases of non-obstructive azoospermia (NOA) to identify the causative factors. Cases $(n=111)$ of NOA referred to the cytogenetics and molecular genetics laboratory of the All India Institute of Medical Sciences in -Patna from 2013-2018 were subjected to 1) karyotyping using GTG bandings techniques, 2) fluorescence in situ hybridization (FISH) for the sex determining region (SRY), and 3) PCR based analysis of STS markers based on microdeletion of the Y-chromosome after isolation of genomic DNA from whole blood. A flow cytometer was used for a cell- kinetic and DNA methylation study after incorporation of 5-azacytidine (5AzaC) $(1.0 \mathrm{ug} / \mathrm{mL})$ in lymphocyte culture. PCR products were analyzed on an agarose gel (1.5\%) and bands were visualized on Gel Doc after ethidium bromide staining. Chromosomal abnormalities, including structural numerical variations, were observed in 14 of the karyotypes. Eight cases showed a 46,XY/47,XYY i.e. mosaic pattern; two cases $46, \mathrm{XY} / 45 / \mathrm{XO}$; a single case with $47, \mathrm{XY}+16$; two cases with $46, \mathrm{X}+$ ring $\mathrm{Y}$; a single case with $46, \mathrm{XY}+$ dicentric in
\end{abstract}


chromosome-7; and two cases showed a normal 46,XY karyotypic pattern. Cellular proliferation increased after incorporation of 5-azaC $(1.0 \mu \mathrm{g} / \mathrm{mL})$ for $24 \mathrm{~h}$ in lymphocyte culture at the $\mathrm{S}$ - phase of the cell cycle. Out of 14 cases analyzed with FISH, three cases showed loss of the SRY region, amongst which two cases were SRY negative (ve) with a normal 46, XY karyotype. Serum follicle stimulating hormone values were higher $(21 \mathrm{U} / \mathrm{L})$ in cases of mosaicism as compared to normal individuals. The frequency of microdeletion deletion of the AZFc region varied in different cases; one case of infertility showing deletion of the Sy 267 STS marker (102bp); loss of a 350bp band (Sy 254) was found in all the cases of infertility. These genetic variations are responsible for dysregulation of spermatogenesis leading to infertility in males. Genetic counselling would be relevant in such cases, before beginning assisted reproductive techniques such as in vitro fertilization.

Key words: Infertility; FISH analysis; SRY probe

\section{INTRODUCTION}

Infertility is a serious reproductive failure affecting approximately $15 \%$ of couples worldwide; the male factor alone contributes to $50 \%$ of the involuntarily childless couples (Hamada et al., 2013; Agarwal et al., 2015). Also, more than 25\% cases of infertility are unable to find the etiopathology and are categorized as "unexplained cause" of infertility (Mol et al., 2018). Chromosomal abnormalities are one of the most relevant causes of primary infertility, in which 5 to $14 \%$ cases are associated with clinical conditions. On the basis of semen analysis, the cases can be classified as either oligozoospermic or azoospermic with aneuploidy of 47, XXY in the karyotype (Tiepolo et al., 1976; Hamada et al., 2013; Colaco and Modi, 2018; Laan, 2019; Oud et al., 2019). Klinefelter syndrome (KFS) constitutes the largest group (15\%) classified as mosaic with a 46, XY/47, XXY karyotype leading to gonadal dysgenesis. Clinically the cases of KFS are characterized by hypotrophy of the testis, azoospermia, increased plasma follicle stimulating hormone (FSH) levels, low testosterone levels, low I.Q. and abnormal phenotype (Ratcliffe et al., 2014). The extra copy of Y- chromosome having karyotype 47, XYY falls in the rare category of KFS with variable phenotype and aggressive behavior in such patients (Robinson and Jacobs, 1999; Ross et al., 2012). The microdeletion of the Y-chromosome in karyotypes of $47, \mathrm{XXY}$ or mosaic $46, \mathrm{XY} / 47, \mathrm{XXY}$ cases with clinical features characterized by testicular hypotrophy, azoospermia. Azoospermic cases with karyotype 46, XX (46, XX maleness, SRY positive XX male) are rare and they are consistently sterile in nature. Clinically, these cases have mature male genitalia, normal height and unimpaired intelligence (Majzoub et al., 2017).

Imbalance (5\%) of the $\mathrm{Y}$-chromosome has been reported in cases of oligozoospermia with abnormal seminograms (Olesen et al., 2001). Mostly, the distal region of Y-chromosome is translocated to the short arm of an acrocentric chromosome using fluorescence in situ hybridization (FISH), which seems to be relevant for diagnosis of microdeletion of Y-chromosome in karyotypes of 47, XXY or mosaic 46, XY/47, XXY 
cases with clinical features characterized by testicular hypotrophy in azoospermic cases. A large number of genetic factors including microdeletion of $\mathrm{Y}$ - chromosome regions (AZF) are known to play crucial roles during spermatogenesis in male infertility (Vogt et al., 1996; Pandey et al., 2013; Yu et al., 2015; Saxena and Kumar, 2016; Saxena et al., 2018; Saxena et al., 2019). The sex determining region SRY gene plays a crucial role in testis determining factor (TDF) located on euchromatic region of Y-chromosome and approximately $80 \%$ cases are SRY- positive with normal phenotype having 46,XY karyotypes (Wu et al., 2014; Majzoub et al., 2017; Mohammadpour et al., 2017). Our objective was to investigate 1) cytogenetic abnormalities and their association with the SRY region in variable karyotypes; and, 2) to evaluate microdeletion of the $\mathrm{Y}$ chromosome using STS makers to understand the genetic basis of spermatogenesis in azoospermic cases.

\section{MATERIAL AND METHODS}

Our study included clinically diagnosed cases $(n=111)$ of non-obstructive azoospermia (NOA) referred to the Cytogenetic and Molecular laboratory, Department of Pathology/Lab Medicine, AIIMS-Patna for genetic analysis. Family history of the infertile couples was recorded to develop pedigree analysis and to find out the mode of inheritance of the mutant allele to the proband. Azoospermia was defined as total absence of sperms in ejaculated semen. Family history showing lack of environmental exposure or exposure to radiation or prenatal exposure to drug that could account for their infertility. Median age of the proband was 33.7 years (range $25-53$ years). Blood samples $(2.0 \mathrm{~mL})$ were collected into a heparinized sterile vial. The patients gave informed written consent for cytogenetic analysis. The study was approved by the institutional ethics committee.

\section{Cytogenetic analysis with GTG banding for Chromosome and SRY region by Fluorescence in Situ Hybridization.}

Karyotypes was developed after collection of $1.0 \mathrm{~mL}$ of blood from proband and short term lymphocytes cultures were set up for $72 \mathrm{~h}$ at $37^{\circ} \mathrm{C}$, using RPMI 1640 media. Phytohemagglutinin was used for stimulation with 5\% FBS (Fetal Bovine Serum) and antibiotics. Before harvesting of cultures, colchicine was added $2 \mathrm{~h}$ prior to arrest the dividing cells, $\mathrm{KCl}$ solution was used as a hypotonic and cells were fixed using 3:1 methanol: acetic acid. At least 50 well spread metaphases were selected to develop karyotypes after GTG banding (Seabright, 1971) .The karyotypes were prepared according to the recommendations of the International System for Chromosome Nomenclature (ISCN 2013) using applied spectral imaging software (Genesis USA) (Simons et al., 2013). 5AzaC $(1 \mu \mathrm{g} / \mathrm{mL})$ act as DNA inducer in the lymphocyte was added to the cultures to increase the cell proliferation rate (mitotic index) and to improve the quality of chromosome morphology since the infertile idiopathic males have poor endocrine function and slow cell proliferation index (Saxena et al., 2007). Flowcytometry analysis was performed for the study of cell - kinetics and the $1 \times 10^{6}$ cells (lymphocytes) were collected from cultures (Webster et al., 1995; Darzynkiewicz et al., 2010). The lysis of red blood cells was induced by the addition of $\mathrm{RBC}$ lysis buffer for $10 \mathrm{~min}$ at $37^{\circ} \mathrm{C}$ and spun at $900 \mathrm{x}$ rpm for $5 \mathrm{~min}$. The cells were washed twice by centrifugation in phosphate-buffered saline (PBS) at $500 \mathrm{~g}$ for 5 min. Then, $10^{6}$ cells were permeabilized with $1.0 \mathrm{~mL}$ of ice cold ethanol $\left(1 \mathrm{~h}, 4^{\circ} \mathrm{C}\right)$ followed 
by washing with PBS and staining with propidium iodide (PI) for 30 min. The lymphocytes were sorted based on forward and side scatter on flowcytometer FACS Canto-II (BD Bioscience, Heidelberg, Germany). PI used as fluorescence dye for cell- cycle analysis to confirm the cell proliferation kinetics.

FISH analysis was carried out for the study of SRY specific region of X and Y chromosome in both interphase as well as metaphases using FISH probe obtained from Abbott-Vysis, Inc. (USA). X- Chromosome identified by green signal and Y-chromosome SRY Spectrum Orange/CEP X Spectrum Green (Abbot Molecular, USA).

\section{Isolation of DNA and PCR}

Genomic DNA was extracted according to standard procedure from peripheral blood samples according to the manufacturer's recommendations (Promega kit USA). DNA contents were measured by Nanodrop spectrophotometer (Thermo USA). PCR was performed to analyse the microdeletions in the AZF region of the Y-chromosome. Each patient was analysed for the presence of specific sequences tagged sites (STS markers Sy 254 \& Sy267) primers $\mathrm{F}^{5}$ GGG TGT TAC CAG AAG CTT GCA AA R GAA CCG TAT CTA CCA AAG CAG $\mathrm{C}^{3}$ and F 5'GAA TGT GTA TTC AAG GAC TTC TCG and R' TAC TTC CCT CGG GGC CTC T 3', respectively of AZFc region.PCR amplification will be performed $25 \mu 1$ reaction volume containing 10x Tris $(\mathrm{pH} 8.4), 50 \mathrm{mM} \mathrm{KCl}, 25 \mathrm{mM}$ $\mathrm{MgCl}_{2}, 2.5 \mathrm{mM} \mathrm{dNTP}, 10 \mathrm{pM}$ of oligonucleotides primers, 50 - $100 \mathrm{ng}$ DNA and $1 \mathrm{U}$ Taq DNA polymerase and PCR condition were $95^{\circ} \mathrm{C}-5 \min \left(94^{0}-30 \mathrm{~s}, 56^{\circ} \mathrm{C}-45 \mathrm{~s}, 72^{0}-45 \mathrm{~s}\right) \mathrm{X}$ 35 cycles, $72^{\circ} \mathrm{C}-5 \mathrm{~min}$ for SY $254(350 \mathrm{bp})$ and $95^{\circ} \mathrm{C}-50\left(94^{0}-30 \mathrm{~s}, 56^{\circ} \mathrm{C}-45 \mathrm{~s}, 72^{0} \mathrm{C}-45 \mathrm{~s}\right)$ X 35 cycles, $72^{\circ} \mathrm{C}-5 \mathrm{~min}$ for SY $267(102)$ bp, respectively. PCR product (bands) was analyzed on agarose (1.5\%) gel and visualized by Gel Doc system (Bio Rad, USA) after Etbr staining.

\section{Hormonal investigations}

Endocrine dysfunction is more prevalent in infertile men than in the general population. Hormonal screening becomes relevant to determining follicle stimulating hormone (FSH), luteinizing hormone (LH) and testosterone levels in case of male infertility. Blood serum sample from the infertile patients will be used for the study hormones assay using standard routine laboratory procedure (ELISA).

\section{Statistical analysis}

Chi square test were apply to find significance difference between cases and their respective controls.

\section{RESULTS}

Based on review of the literature, proposed mechanism of interchromosomal translocation is difficult to explain in the proband. The etiopathology of SRY-negative and their frequency in all the 14 cases of non-obstructive azoospermic male with karyotypic 
variations $46, \mathrm{XY}, 47, \mathrm{XY}+16,46, \mathrm{XY}$ ring chromosome of $\mathrm{Y}$ showed a high frequency of mosaicism (46,XY/47,XYY) (Table 1).

Table 1. Karyotypic variations and SRY analysis in cases of male infertility.

\begin{tabular}{|c|c|c|c|c|c|c|}
\hline No. & $\begin{array}{l}\text { Age } \\
(\mathbf{y r})\end{array}$ & $\begin{array}{l}\text { Case } \\
\text { ID. }\end{array}$ & $\begin{array}{l}\text { FSH profile } \\
\text { (U/L) }\end{array}$ & $\begin{array}{l}\text { FISH } \\
\text { (SRY) }\end{array}$ & Karyotypes & References \\
\hline 1 & 37 & 382 & 4.13 & + & $46, \mathrm{XY}$ & Mierla et al. 2014 \\
\hline 2 & 32 & 141 & 6.03 & + & $46, X Y / 47, X Y Y$ & Sadik and Seifeldin (2014) \\
\hline 3 & 34 & 140 & 3.02 & + & $\begin{array}{l}46, X Y / 47, X Y Y \\
46, X(r) Y \\
45, X 0\end{array}$ & $\begin{array}{l}\text { Pylyp et al. 2013; Pylyp et al. 2015; Carvalho et } \\
\text { al. } 2007\end{array}$ \\
\hline 4 & 52 & 99 & 16.4 & - & $46, X Y$ & Harton and Tempest 2012 \\
\hline 5 & 33 & 85 & 18.7 & + & $46, \mathrm{XY} / 47, \mathrm{XYY}$ & Bhasin et al. 1994 \\
\hline 6 & 25 & 01 & 20.84 & + & $46, \mathrm{XY} / 47, \mathrm{XYY}$ & Pylyp et al. 2015; Kim et al. 2017 \\
\hline 7 & 30 & 122 & 2.16 & - & $46, X Y$ & Harton and Tempest 2012 \\
\hline 8 & 33 & 195 & 9.07 & + & $\begin{array}{l}46, \mathrm{XY} / 47, \mathrm{XYY} \\
45, \mathrm{XO}\end{array}$ & $\begin{array}{l}\text { Bulakbasi T et al. } 2008 \\
\text { Clementini et al. } 2005\end{array}$ \\
\hline 9 & 35 & 134 & 16.14 & + & $46, \mathrm{XY} / 47, \mathrm{XYY}$ & Bhasin et al. 1994 \\
\hline 10 & 25 & 135 & 8.3 & - & $47, \mathrm{XY},+16$ & Wolstenholme et al. 1995 \\
\hline 11 & 43 & 121 & 14.01 & + & $46, X Y / 47, X Y Y$ & Bhasin et al. 1994, Morel et al. 1999 \\
\hline 12 & 29 & 163 & 6.13 & + & $46, X Y, \operatorname{dic}(7),(r) Y$ & Carvalho et al. 2007 \\
\hline 13 & 33 & 139 & 13.4 & + & $46, X Y / 47, X Y Y$ & Bhasin et al. 1994; Robinson and Jacobs, 1999 \\
\hline
\end{tabular}

* At least 50 well spread metaphase plates were selected for karyotyping to find structural and numerical variations according to ISCN 2013. ** FSH normal range: 1.5-12.4 U/L

The 14 cases of male infertility had a median age of 33.4 years (range 25 - 52 years) referred for cytogenetic and molecular genetic analysis to evaluate stable and unstable interchromosomal variations. These cases showed normal phenotypic characteristic features that included presence of external genitalia, medium stature with normal behavior. Figure 1 shows the pedigree analysis, which revealed a paternal mode of inheritance based on Mendel's law following either law of segregation of alleles or independent assortment to arrive at the proband in heterozygous condition. The other families showed a lack of family history with chromosomal abnormalities, i.e. structural (translocation) and numerical (aneuploidy), suggesting that these mutations in the proband are sporadic in nature.

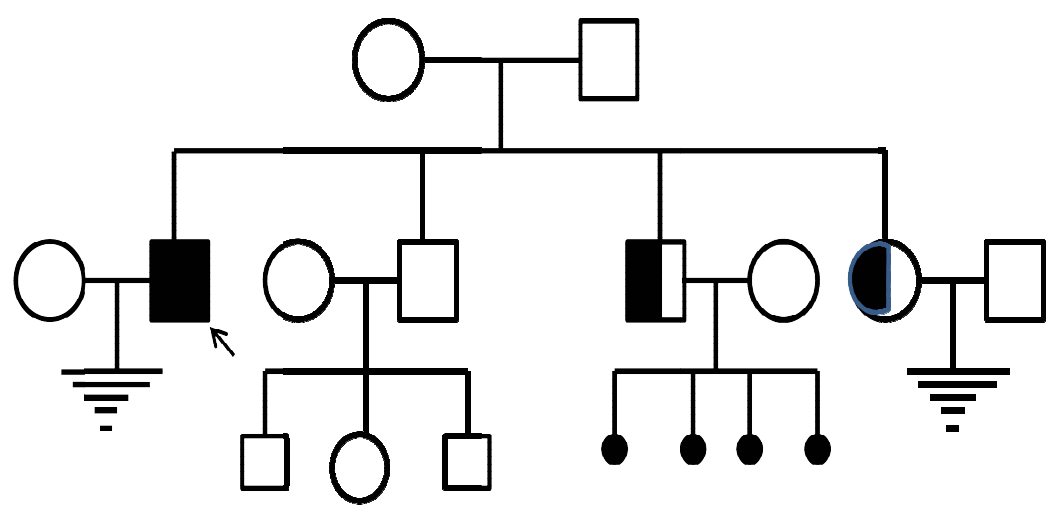

Figure 1. Pedigree analysis (representative) showing the severity of disease (infertility) in a proband (arrow) with a complex paternal mode of inheritance based on Mendel's principals either following the law of segregation of alleles or independent assortment. 
In order to perform chromosomal studies using short term lymphocyte cultures, flow cytometry was also carried out to evaluate the proliferation of cells (lymphocytes) based on forward scattering (FSC) and side scattering (SSC) followed by analysis of the DNA content and cell - cycle analysis as depicted in Figure 2.
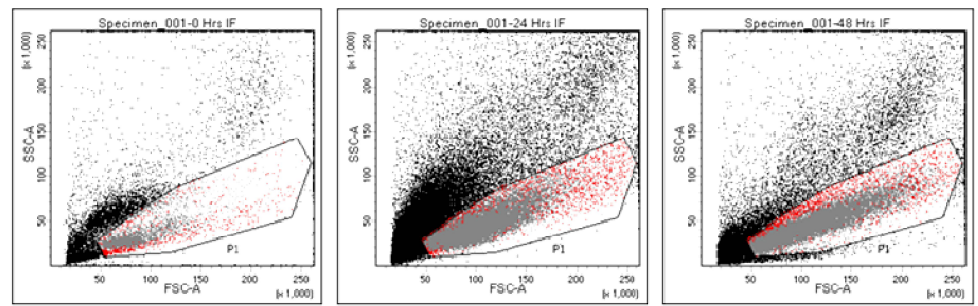

Change in Morphology of the Lymphocytes with different culture time

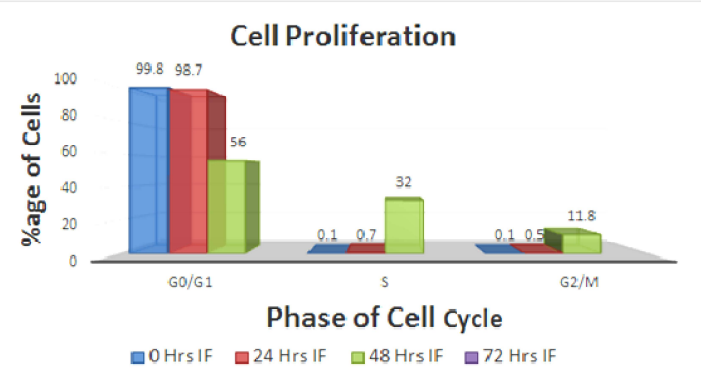

Figure- 2A. In-vitro analysis of light scatter characteristic of normal human lymphocytes showing erythrocyte lysis. Forward Scatter (FCS abscissa) showing cell size and side scatter (SSC; coordinate) indicate the cell granularity. The light scatter regions showing the location of polymorph nuclear cells (PMN), monocytes (M), and lymphocyte cell population after using propidium iodide (PI) as a fluorescenct dye for flow cytometry analysis. The histogram was prepared against different phases of cell - cycle analysis i.e. G0/G1, S and G2 phases of cellular proliferation of human lymphocytes in a male infertility case.

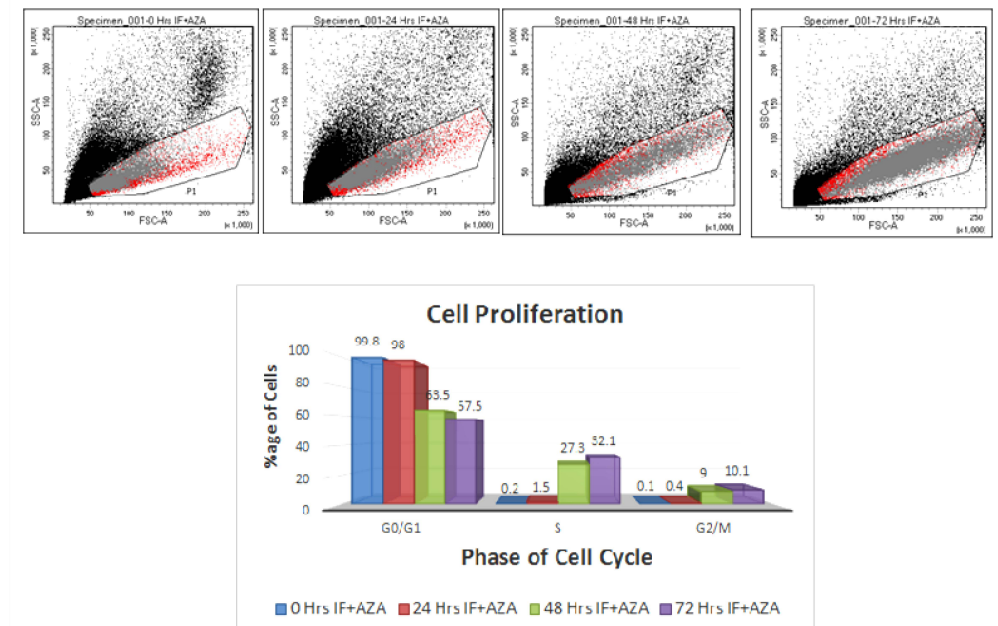

Figure-2B. Flow cytometry analysis of cellular proliferation of human lymphocytes after incorporation of 5-AzaC (1.0 $\mu \mathrm{g} / \mathrm{mL}$ ) showing forward scattering (FSC) and side scattering (SSC) based of sorting of PI stained cells. Histogram showing different phases of cell-cycle analysis at G0/G1, S and G2 phases in lymphocyte culture at 24, 48 and $72 \mathrm{~h}$.

Genetics and Molecular Research 18 (2): gmr18349

CFUNPEC-RP www.funpecrp.com.br 
The cells were treated with or without 5-AzaC, proliferation rate increase at $24 \mathrm{~h}$ to $48 \mathrm{~h}$ of the cells treated with 5-AzaC during S phase of the cell - cycle as compared G1/G2 phase and controls. Table 2 reveals the detail individual frequency of the structural and numerical chromosomal variations, the highest frequency $(7 \%)$ was observed in eight cases (7\%) with 46,XY/47,XYY i.e. mosaic pattern; two cases (1.8\%) 46, XY/45/XO; single case $(0.9 \%)$ with $47, \mathrm{XY}+16$; two cases $(1.8 \%)$ with $46, \mathrm{X}(\mathrm{r}) \mathrm{Y}$; single case $(0.9 \%)$ with $46, \mathrm{XY}+\operatorname{dic}(7)$; and two cases (1.8\%) with 46,XY karyotypic pattern.

Table 2. Karyotypic changes and their frequency in 111 cases of infertility in India.

\begin{tabular}{lll}
\hline S.No. & Chromosomal Variations & Number of cases \\
\hline 1 & $46, \mathrm{XY} / 47, \mathrm{XYY}$ & 8 \\
2 & $46, \mathrm{XY} / 45, \mathrm{X} 0$ & 2 \\
3 & $46, \mathrm{X}(\mathrm{r}) \mathrm{Y}$ & 2 \\
4 & $47, \mathrm{XY},+16$ & 1 \\
5 & $46, \mathrm{XY},+\operatorname{dic}(7)$ & 1 \\
\hline
\end{tabular}

In the present study three cases shows loss of SRY locus (21\%), but two cases (1.8\%) showing SRY negative with 46, XY (normal karyotype) as depicted in Figure 3.
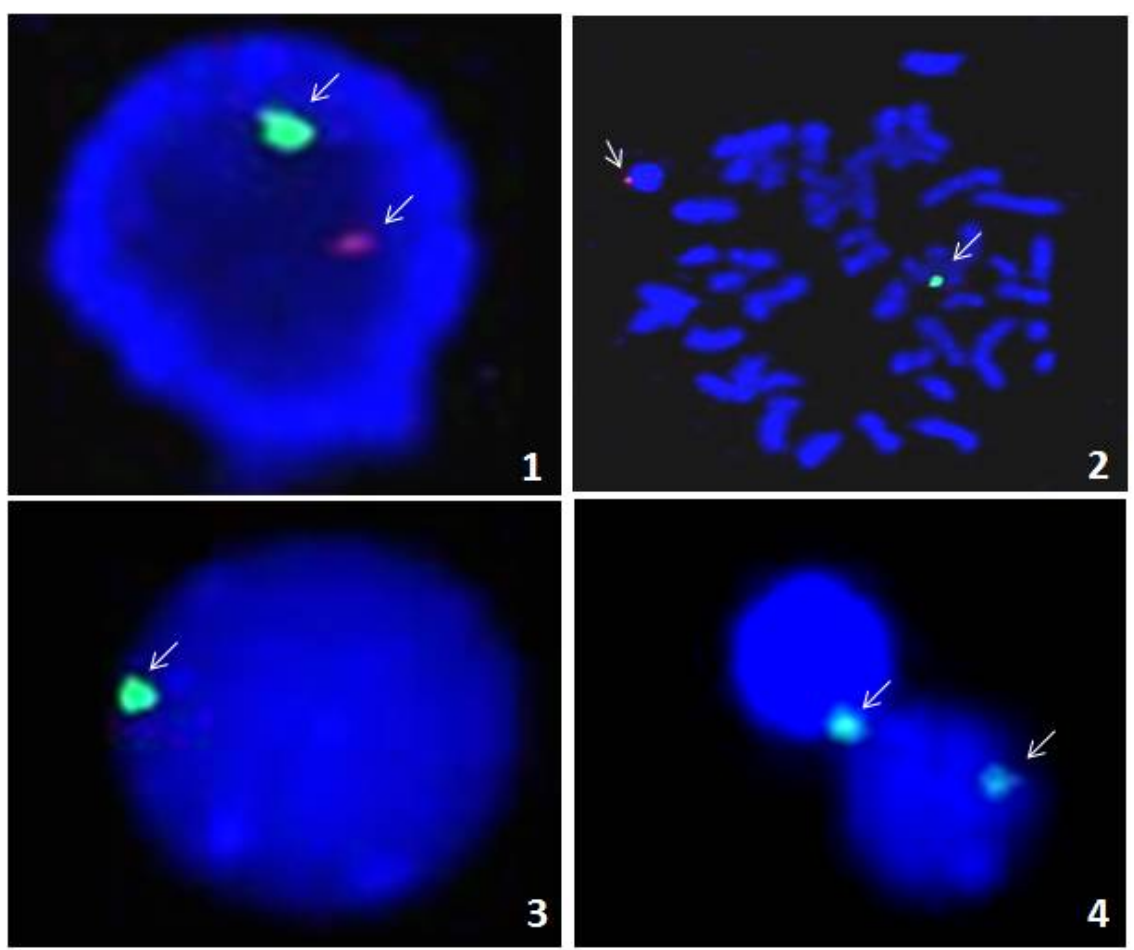

Figure 3A. Proband showing loss of SRY region in the case of an azoospermic male. Interphase cells showing positive signal of SRY+ve probe (orange) and X chromosome ((CEP X green) in the periphery of the cells, DAPI (blue) used for DNA staining (1). Metaphase plate and Y-chromosome showing of SRY+ve probe (orange) and green signal appears on chromosome $\mathrm{X}$ (2). Interphase cells showing lack of signal of SRY-probe (orange) in of an azoospermic male with presence of an $\mathrm{X}$ chromosome showing a green signal (Figure 3,4 ). 

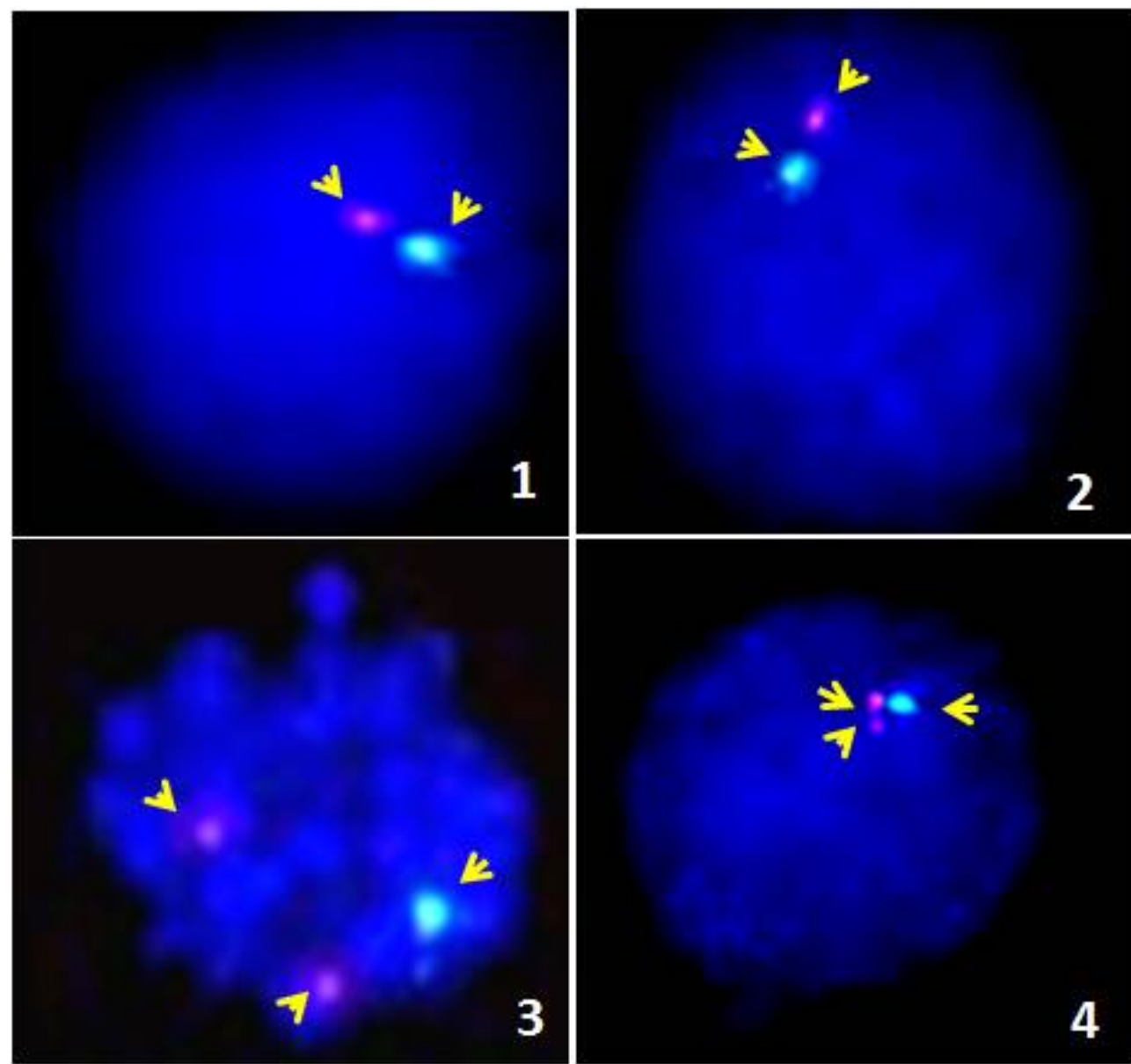

Figure 3B. Identification of sex chromosomes (X and Y) using FISH analysis. Nuclei were hybridized with Ychromosome (orange) and X chromosome (CEP X green) using a Vysis probe (USA) and DAPI (blue) used for DNA staining of the periphery of the cell. FISH analysis showing $X$ and $Y$ chromosome (normal 1,2) and an extra copy of the Y-chromosome in interphase and prometaphase $(3,4)$ in an infertile case.

FISH analysis also confirmed the extra of Y-chromosomes, after using CEP Y probe with orange spectrum in the mosaic cases having karyotypes 46, XY/47XYY as shown in Figure 4.

The median value in serum FSH level shows $10.64 \mathrm{mLU} / \mathrm{mL}$ as compared to normal $2.5-14.0 \mathrm{~mL} \mathrm{U} / \mathrm{mL}$ with highest value in mosaic cases. Molecular genetic analysis revealed the loss of two locus SY 267 and SY 350 belongs to euchromatic region of Y-Chromosome i.e. AZFc in one cases (7\%) of 102bp (SY267), and 350bp (Sy 254) band was deleted in all the cases of NOA. 


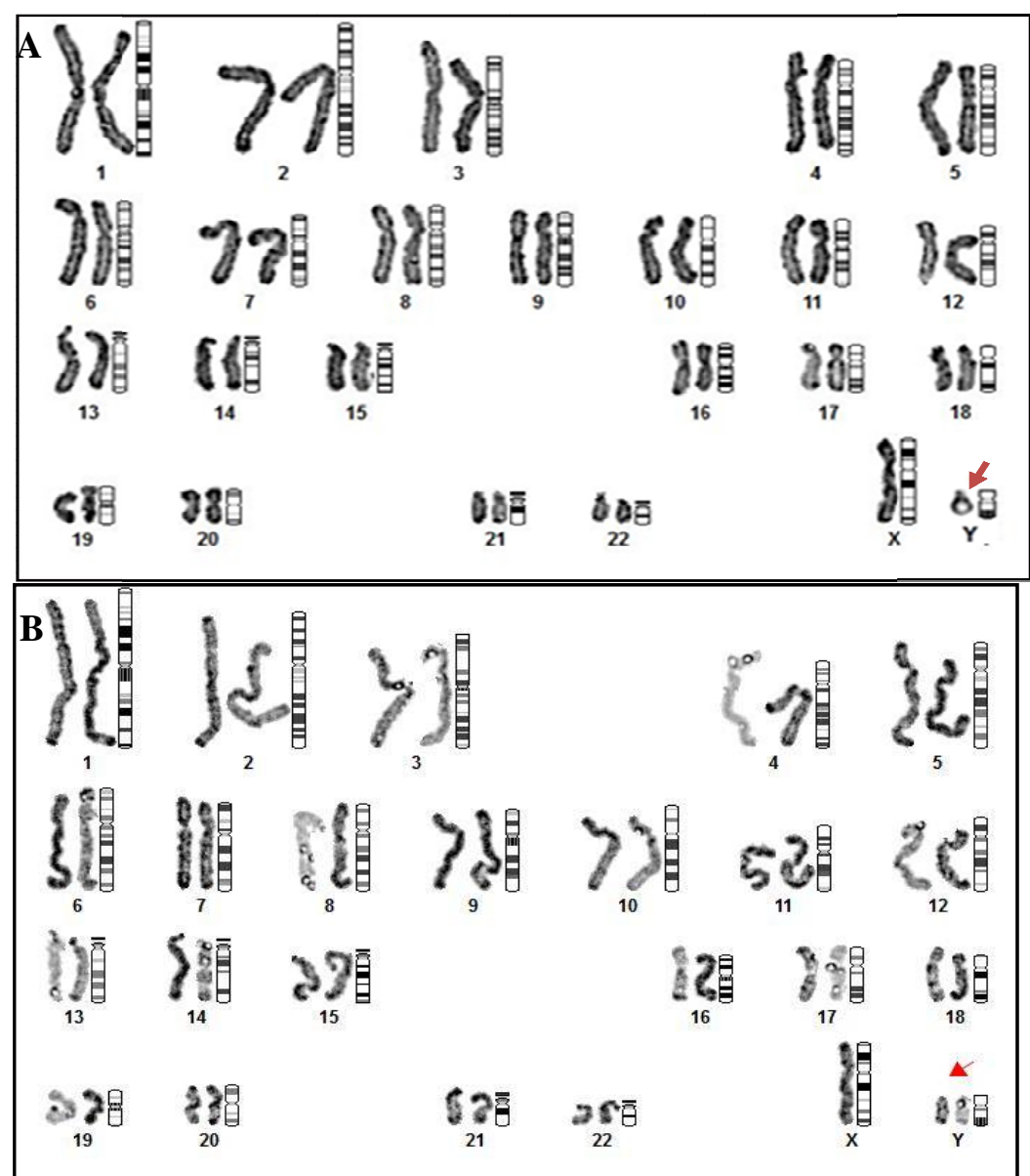

Figure 4A. Karyotype showing the 46,XY with ring (Y) in infertile male after GTG -banding of chromosome according to ICSN 2013. 4B: Karyotype showing the extra copy of Y chromosome, 47,XYY, after GTG -banding of chromosome according to ICSN 2013.

\section{DISCUSSION}

Genetic causes of male infertility in human has been well recognized in different karyotypes with variation in the frequency which varies from 5\% to $15 \%$ in different clinical cases of azoospermia (Martin, 2008). The imbalance of Y-chromosome has (5\%) been reported in the cases of oligozoospermia with abnormal seminograms (Olesen et al., 2001). In most of the cases, the distal region of Y- chromosome is translocated to the short arm of an acrocentric chromosome and visualized by fluorescence in situ hybridization seems to be relevant for diagnose of microdeletion of Y-chromosome in karyotypes of 47, XXY or mosaic 46,XY/47,XXY cases with clinical features characterized by testicular hypotrophy, azoospermia in syndromic cases. The expected phenotypic variation from oligozoospermia to azoospermia has variable impact on karyotype within the same family (Foresta et al., 2002). FISH have an advantage for larger sample size to evaluate the interchromosomal effect i.e. Rebertsonian translocation or reciprocal translocation during segregation of chromosomes. Large number of genetic factors including microdeletion of euchromatic region of Y-chromosome i.e. AZFa,b,c 
are known to play crucial role in NOA cases (Ambulkar et al., 2014; Saxena et al., 2019). The deletion frequency AZF regions varying in azoospermic cases due to different genetic factors are known to play crucial role during spermatogenesis (de Sousa Filho et al., 2018; Goncalves et al., 2017). Although, there is no correlation exist between genotypic-phenotypic variation and deletion frequency of AZF regions varying in azoospermic cases (Saxena et al., 2019).

The spermatogenesis is highly complex phenomenon leading to interfare with male infertility. The chromosomal abnormalities are one of the relevant features and the exact cause of male infertility is still difficult to define but more than $50 \%$ cases are affected due to reproductive dysfunction (Martin, 2008; Mierla et al., 2014). Several studies have shown that the frequency of chromosomal abnormalities increases from $10-15 \%$ in azoospermic cases due to variations in the environmental factors. Amongst, $\sim 5 \%$ are associated with either numerical or structural abnormalities and $\sim 2 \%$ are mosaic with autosomal abnormalities. Most common abnormality in azoospermic cases (15\%) are the presence of extra copy of Y-chromosomes leading to develop variation in karyotypes 47, XXY (aneuploidy) or 47, XYY chromosomes with different mode of inheritance to the male proband (Robinson and Jacobs, 1999; Ross et al., 2012). The sex determination events are regulated by Y-chromosome and its gene content by either losing or gaining sequences through the action of various actions including recombination mutation, insertion and transposition (Robinson and Jacobs, 1999; Goncalves et al., 2017). Many studies have been documented the frequency of chromosomal aberrations from $2.2-16 \%$ in male infertility (Mol et al., 2018; Laan, 2019; Oud et al., 2019), but present study shows the variations between $0.9 \%$ to $7 \%$ in NOA cases with the maximum frequency (7\%) in the cases of mosaic 46,XY/ 47, XYY cases. Probably, this occurs due to meiotic non- disjunction event of Xchromosome or in anaphase lag of chromosome from a normal 46,XY or 47, XXY zygote, suggesting loss of meiotic cells at pachytene stage which increase the risk of developing aneuploid sperm. Present study further confirm the event of chromosomal segregation as observed in earlier study in our laboratory during incorporation of 5-AzaC in cell culture (Saxena, 2007). The light scattering is the characteristic feature of peripheral lymphocyte subset and there are three major subset of cell population are polymorphic cells, monocytes and lymphocytes in normal peripheral blood visualized due to erythrocyte lysis based on the light scattering as shown in Figure 2A. This study is based on the characteristic features of lymphocytes based on light scattering i.e. FSC and SSC properties for sorting of the cells in the gated population. Such gated population is analyzed for different phases of cell- cycle using PI as fluorescence dye for DNA staining. DNA methylation study help after incorporation of 5AzaC becomes valuable technique for cell - cycle kinetics in the case of idiopathic infertile males, where the proliferation rate of cells (lymphocytes) becomes slow and showing low mitotic index (MI). However, the maximum fluorescence was observed in G0/G1 phase followed by $\mathrm{S}$ phase of the cell - cycle with the limitation of this study should be further extended in other laboratories to understand the germ cell - kinetics due to sporadic mutation in male infertility. These chromosomal abnormalities are responsible for failure of spermatogenic and reduction of testis (size) causing azoospermia and clinically develop as Klinefelter syndrome. Interestingly, present study reveals high frequency (7\%) of mosaicism with 46XY/47, XYY karyotype, whereas, in South Indian population shown very low frequency i.e. 0.5\% (Sreenivasa et al., 2013). The reason in the changes in the frequency of mosaicism in two different populations are may be either due to different profession like farmers using chemical fertilizers / pesticides or truck drivers who are expose to automobile fumes that may lead to nondisjunction event during meiosis or may be due to unidentified environmental factors.

Previous studies by Yoshida et al. (1997) have suggested in Klinefelter syndrome and 47XYY karyotypes have 50\% sperms carry extra copy of Y- chromosome, thus increasing the 
"risk" of male infertility. The frequency of deletion regions is quite variable in different populations due to different environmental factors (Krausz et al., 2003, 2014). The AZFa and $\mathrm{AZFb}$ deletions are mostly associated with Sertoli-cell-only syndrome and arrest of spermatogenesis, respectively (Krausz et al., 2001). The present study shows the deletion of $\mathrm{AZFc}$ region of $102 \mathrm{bp}$ (SY267) with high frequency (7\%) due to regional variation in existing population that includes changes in the life-style.

In earlier study, a case of SRY-negative 46, XX male with mature male gonads with male infertility has been reported by Rajendra et al. (2006). Interestingly, present study also shows variation in the expression of SRY, three cases of SRY negative were observed with two normal karyotype 46,XY. The inter and intra variation of chromosome aberration are associated with various clinical manifestation including cancer and infertility. There is positive correlation were observed between $A Z F \mathrm{c}$ deletion and variation in structural abnormalities $46, \mathrm{X}(\mathrm{r}) \mathrm{Y}$; $46, \mathrm{XY}, \operatorname{dic}(7)$ as well as numerical variation having extra copy of $\mathrm{Y}$ - chromosomes developing mosaicism 46,XY/47,XYY karyotypes chromosome abnormalities (Clementini et al., 2005; Mierla et al., 2014). However, the present study shows a frequency $(0.9 \%)$ of dicentric chromosomes involving chromosome -7 probably due to lag in anaphase and presence of two centromeres might have disturbed normal segregation of chromosomes in mitosis and has not been reported earlier in the cases of male infertility. Two cases showed high frequency (1.8\%) of ring of Y chromosome and proposed mechanism of such formation with involvement of AZF regions are responsible for male infertility as depicted in Figure 5.

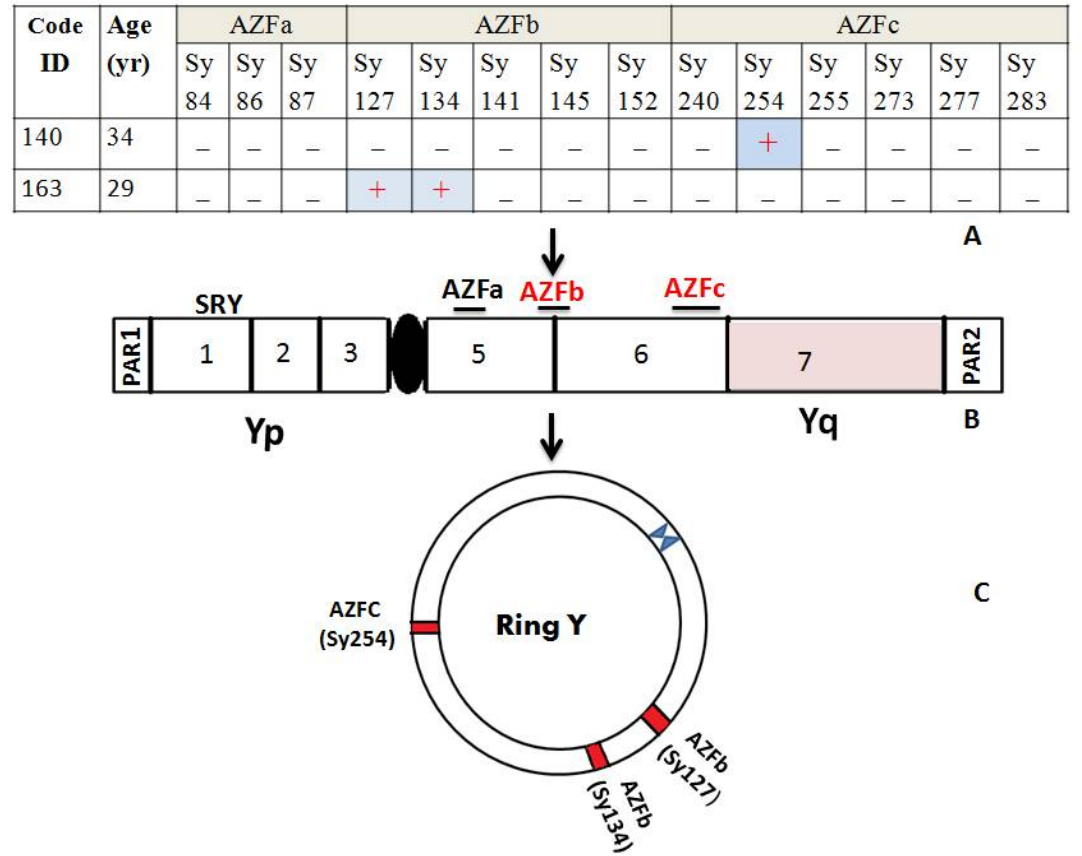

Figure 5. Molecular analysis of formation of Ring of $\mathrm{Y}$ - chromosome., the deletion of long arm of $\mathrm{Y}$ chromosome region AZFb (loss of SY127, SY134) and AZFc (involving loss of SY254) as represented by (+) for presence of mutation and (-) for absence of mutation after PCR based analysis of AZF region (STS markers) as documented in table (A). Schematic representation of Y-chromosome showing three different regions (AZF) regulating spermatogenesis, (B) formation of Ring chromosomes showing the expected molecular basis of mutation after breakage and ligation at break point regions interfare with germ cell proliferation. This diagram is a representation of a molecular version of the ring Ychromosome structure as observed in the karyotype (Figure 4A). 
Such study possible help to explain the putative break point involving euchromatic region including the deletion of AZFb (Sy127, Sy134) and AZFc (Sy267) locus may be involved in gonadal dysgenesis. Such findings may validate one of the relevant causative factors of male infertility. However, there is a lack of correlation exist between loss of SRY region and microdeletion of $\mathrm{Y}$ - chromosome observed in the present study.

\section{CONCLUSIONS}

Genetic factors are quite relevant because they play a significant role in the infertile male population belonging to different professions, including farmers exposed to pesticides and inorganic fertilizers, truck drivers (exposed to automobile fumes) or exposed to radiation leading to DNA damage (gene mutation). We detected both structural and numerical variations, increasing the "risk" of imperfect spermatogenesis. However, the biological complexity of infertile couples requires comprehensive genetic counseling, including social aspects that can help in developing future strategies for assisted reproductive techniques, which include in-vitro fertilization, intra cytoplasmic sperm injections and testicular sperm extraction whenever vertical transmission of AZF mutation is observed; otherwise the problem of infertility continues and falls under the category of an "unexplained" cause.

\section{ACKNOWLEDGMENTS}

AKS thankfully acknowledges Director, AIIMS Patna for valuable suggestions and the DBT project (No.BT/PR14671/MED/12/487/2010) for providing financial assistance to carry out the work.

\section{REFERENCES}

Agarwal A, Mulgund A, Hamada A and Chyatte MR (2015). A unique view on male infertility around the globe. Reprod. Biol. Endocrinol. 26: 13:37.

Ambulkar PS, Sigh R, Reddy M, et al. (2014). Genetic Risk of Azoospermia Factor (AZF) Microdeletions in Idiopathic Cases of Azoospermia and Oligozoospermia in Central Indian Population. J. Clin. Diagn. Res. 8(3): 88-91.

Bhasin S, de Kretser DM and Baker HW (1994). Clinical review 64. Pathophysiology and natural history of male infertility. J. Clin. Endocrinol. Metab. 79: 1525-9.

Bulakbasi T, Sahin F, Yil maz Z and Zeyneloglu H (2008). A 45,X/47,XYY/46,XY Karyotype and Y Chromosome Microdeletion in an Infertile Male. Balkan J. Med. Gen. 11(1): 51-4.

Carvalho FM, Wolfgramm EV and Degasperi I (2007). Molecular cytogenetic analysis of a ring-Y infertile male patient. Genet. Mol. Res. 6: 59-66.

Clementini E, Palka C, Iezzi I, et al. (2005). Prevalence of chromosomal abnormalities in 2078 infertile couples referred for assisted reproductive techniques. Hum. Reprod. 20: 437-42.

Colaco S and Modi D (2018). Genetics of the human Y chromosome and its association with male infertility. Reprod. Biol. Endocrinol. 16: 14. doi:10.1186/s12958-018-0330-5.

Darzynkiewicz Z, Halicka HD and Zhao H (2010). Analysis of cellular DNA content by flow and laser scanning cytometry. Adv. Exp. Med. Biol. 676: 137-47.

de Sousa Filho EP, Christofolini DM, Barbosa CP, et al. (2018). Y chromosome microdeletions and varicocele as aetiological factors of male infertility: A cross-stional study. Andrologia. 50(3). doi: 10.1111/and.12938.

Foresta C, Moro E and Ferlin A (2001). Y chromosome microdeletions and alterations of spermatogenesis. Endocr. Rev. 22: $226-39$.

Goncalves C, Cunha M, Rocha E, et al. (2017). Y-chromosome microdeletions in nonobstructive azoospermia and severe oligozoospermia. Asian J. Androl. 9: 338-345.

Hamada AJ, Esteves SC and Agarwal A (2013). A comprehensive review of genetics and genetic testing in azoospermia. Clinics (Sao Paulo). 68 Suppl 1: 39-60. 
Harton GL and Tempest HG (2012). Chromosomal disorders and male infertility. Asian J. Androl. 14: 32-9.

Kim SY, Kim HJ, Lee BY, et al. (2017). Y Chromosome Microdeletions in infertile Men with Non-obstructive Azoospermia and Severe Oligozoospermia. J. Reprod. Infertil. 18: 307-315.

Krausz C and Chianese C (2014). Genetic testing and counselling for male infertility. Curr. Opin. Endocrinol. Diabetes Obes. 21: 244-50.

Krausz C, Forti G and Meclraeavey K (2003). The Y-chromosome and male infertility. Int. J. Androl. 26: 70-75.

Krausz C, Rajpert-De Meyts E, Frydelund-Larsen L, et al. (2001). Double-blind Y chromosome microdeletion analysis in men with known sperm parameters and reproductive hormone profiles: microdeletions are specific for spermatogenic failure. J. Clin. Endocrinol. Metab. 86: 2638-42.

Laan M (2019). Systematic review of the monogenetic causes of male infertility: the first step towards diagnostic gene panels in the andrology clinic. Hum. Reprod. 34(5): 783-85.

Majzoub A, Arafa M, Starks C, et al. (2017). 46 XX karyotype during male fertility evaluation; case series and literature review. Asian J. Androl. 19: 168-72.

Martin RH (2008). Cytogenetic determinants of male fertility. Hum. Reprod. Update. 14(4): 379-90.

Mierla D, Jardan D and Stoian V (2014). Chromosomal abnormality in men with impaired spermatogenesis. Int. J. Fertil. Steril. 8: 35-42

Mohammadpour Lashkari F, Totonchi M, et al. (2017). 46,XX males: a case series based on clinical and genetics evaluation. Andrologia. 49: e 12710.

Morel F, Roux C and Bresson JL (1999). Sex chromosome aneuploidies in sperm of 47,XYY men. Arch. Androl. 43: 27 36.

Mol BW, Tjon-Kon-Fat R, Kamphuis E, et al. (2018). Unexplained infertility: Is it over-diagnosed and over-treated? Best Pract. Res. Clin. Obstet. Gynaecol. 53: 20-9.

Olesen C, Hansen C, Bendsen E, et al. (2001) Identification of human candidate genes for male infertility by digital differential display. Mol. Hum. Reprod. 7: 11-20.

Oud MS, Volozonoka L, Smits RM, et al. (2019). A systematic review and standardized clinical validity assessment of male infertility genes. Hum. Reprod. 34: 932-41.

Pandey LK, Pandey S, Gupta J and Saxena AK (2010). Loss of the AZFc region due to a human Y-chromosome microdeletion in infertile male patients. Genet. Mol. Res. 9: 1267-73.

Pylyp LY, Spinenko LO, Verhoglyad NV, et al. (2013). Chromosomal abnormalities in patients with oligozoospermia and non-obstructive azoospermia. J. Assist. Reprod. Genet. 30: 729-32.

Pylyp LY, Spinenko LO, Verhoglyad NV, et al. (2015). Chromosomal abnormalities in patients with infertility. Tsitol. Genet. 49(3): 33-9.

Ratcliffe SG, Read G, Pan H, et al. (1994). Prenatal testosterone levels in XXY and XYY males. Horm. Res. 42: 106-9.

Rajendra S, Rajani V, Gupta N, et al. (2006). SRY-negative 46, XX male with normal genitals, complete masculinization and infertility. Mol. Hum. Reprod. 12: 341-6.

Robinson DO and Jacobs PA (1999). The origin of the extra Y chromosome in males with a 47, XYY karyotype. Hum. Mol. Genet. 8: 2205-9.

Ross JL, Roeltgen DP and Kushner H (2012). Behavioural and social phenotypes in boys with 47,XYY syndrome or 47,XXY Klinefelter syndrome. Pediatrics. 129(4): 769-78.

Sadik DI and Seifeldin NS (2014). Fluorescence in situ hybridisation analysis of sex chromosome in non-obstructive azoospermic men. Andrologia 46: 231-9.

Saxena AK, Agarwal M and Tiwari M (2018). Single nucleotide polymorphism of Arylsulfatase D gene (ARSD) and their association with male infertility. J. Clin. Gen. Genomics. 1: 11-13.

Saxena AK, Tiwari M and Aniket K (2019). Penetrance of de novo mutation of USP9Y and CDH11Y gene in AZF regions of non obstructive azoospermic population in India. Int. J. Current Res. 11: 1373-79.

Saxena AK and Gupta RK (2016). Microdeletion of Y-chromosome associated male infertility-impediment and consequence. J. Basic Clin. Rep. Sci. 5: 57-60.

Saxena AK, Singh G and Srivastava AK (2007). Unexpected segregation of chromosome and common fragile site expression induced by 5-Azacytidine exposure in human lymphocytes culture of Down Syndrome patients. Biomed. Res. 18: 31-34.

Seabright M (1971). A rapid banding technique for human chromosomes. Lancet. 2(7731): 971-2.

Simons A, Shaffer LG and Hastings RJ (2013). Cytogenetic Nomenclature: Changes in the ISCN 2013 Compared to the 2009 Edition. Cytogenet. Genome Res. 141: 1-6.

Sreenivasa G, Malini S, Kumari P and Dutta R (2013). Cytogenetic abnormalities in 200 male infertile cases in the southern region of India. Open J. Genet. 3: 33-37.

Tiepolo L and Zuffardi O (1976). Localization of factors controlling spermatogenesis in the nonfluorescent portion of the human Y chromosome long arm. Hum. Genet. 34: 119-24.

Vogt PH, Edelmann A, Kirsch S, et al. (1996). Human Y chromosome azoospermia factors (AZF) mapped to different subregions in Yq11. Hum. Mol. Genet. 5: 933-43. 
Webster GA, Bowles MJ, Karim MS, et al. (1995). Flow cytometric analysis of peripheral blood lymphocyte subset light scatter characteristics as a means of monitoring the development of rat small bowel allograft rejection. Clin. Exp. Immunol. 100: 536-42.

Wolstenholme J (1995). An audit of trisomy 16 in man. Prenat. Diagn. 15: 109-21.

Wu QY, Li N, Li WW, et al. (2014). Clinical, molecular and cytogenetic analysis of 46, XX testicular disorder of sex development with SRY-positive. BMC. Urol. 14: 70.

Yu XW, Wei ZT, Jiang YT and Zhang SL (2015). Y chromosome azoospermia factor region microdeletions and transmission characteristics in azoospermic and severe oligozoospermic patients. Int. J. Clin. Exp. Med. 8: 1463446. 1 . 